\title{
Decay of Stable Warm-Core Eddies in a Layered Frontal Model
}

\author{
Angelo Rubino* AND KATRIN Hessner + \\ Institut für Meereskunde, Universität Hamburg, Hamburg, Germany \\ PETER BRANDT \\ Institut für Meereskunde, Universität Kiel, Kiel, Germany
}

(Manuscript received 19 September 2000, in final form 14 June 2001)

\begin{abstract}
Aspects of the decay of stable frontal warm-core eddies in the deep ocean are investigated using a new numerical layered "frontal" model that solves the nonlinear, reduced-gravity, shallow-water equations for a horizontally inhomogeneous, viscous fluid on an $f$ plane. After a discussion on aspects of the numerical techniques implemented to allow for the eddy expansions and contractions at the sea surface, for the first time the capability of a numerical model of reproducing the evolution of analytical nonstationary frontal vortices is explored. This step is necessary, as far as different phenomena related to the dynamics of these oceanic features are to be studied numerically. In fact the comparison between numerical and analytical inviscid solutions allows for a quantification of the numerical dissipation affecting the simulated solutions. This dissipation is found to be very small in this numerical model: The simulated lifetimes are larger than those of most of the frontal eddies observed in the World Ocean. On this basis, the eddy decay due to interfacial (linear and quadratic) friction, harmonic horizontal momentum diffusion, as well as linear ambient-water entrainment is investigated. It is found that interfacial friction represents a much more efficient mechanism than horizontal diffusion and water entrainment in inducing the eddy decay as well as in damping the eddy pulsations. It is thus suggested that internal wave radiation due to vortex pulsation can represent a relevant mechanism for the dissipation of the vortex energy in a stratified ambient ocean only episodically. Finally, a critical discussion about the appropriateness of the different approximations assumed in the investigation is presented. In particular, the appropriateness of the reduced-gravity assumption is discussed. Results are consistent with those obtained analytically in the frame of the frontal-geostrophic theory: Although the effect of an active ambient layer on the vortex dynamics is found to be virtually absent only for unrealistically large water depths, it appears that the reduced-gravity model describes warm-core eddies acceptably for values of the ratio between maximum vortex thickness and total water depth typical for Gulf Stream rings.
\end{abstract}

\section{Introduction}

Among the most energetic features characterizing the mesoscale circulation in the near-surface ocean are frontal, warm-core eddies. These anticyclonic vortices, which are often characterized by a large stability (they have been often observed to preserve their coherence for several months or even years) consist of rotating masses of anomalous (lighter than the ambient) water that, at the sea surface, are separated from the surrounding water by a closed frontal line. In the last three decades, owing particularly to the advent of satellite oceanography, the

\footnotetext{
* Current affiliation: Dipartimento di Scienze Ambientali dell'Università Ca' Foscari di Venezia, Venice, Italy.

+ Current affiliation: OceanWaveS GmbH, Lüneburg, Germany.
}

Corresponding author address: Angelo Rubino, Institut für Meereskunde, Universität Hamburg, Troplowitzstr. 7, 22529 Hamburg, Germany.

E-mail: rubino@ifm.uni-hamburg.de existence of frontal, warm-core eddies was revealed in all of the World Ocean, their most famous representatives being perhaps the warm core rings released from Gulf Stream meanders (Saunders 1971; Csanady 1979; Evans et al. 1985; Joyce 1984). Typically these eddies are characterized by almost circular surface areas, surface radii of 2-4 times the internal Rossby radius of deformation, maximum thicknesses of 300-600 m, and maximum horizontal velocities of 1-2 $\mathrm{m} \mathrm{s}^{-1}$ (Saunders 1971; Andrews and Scully-Power 1976; Joyce 1984). As frontal, warmcore eddies may contribute substantially to the transport of temperature, salt, and momentum, as well as chemical and biological properties across frontal zones, they are thought to exert a significant influence on the heat, energy, and ecological equilibrium of large oceanic regions (Olson 1991). The large amount of existing observational data showing aspects of the dynamics of frontal, warmcore eddies finds its counterpart in a great number of laboratory experiments and theoretical studies devoted at understanding the general nature of this phenomenon 


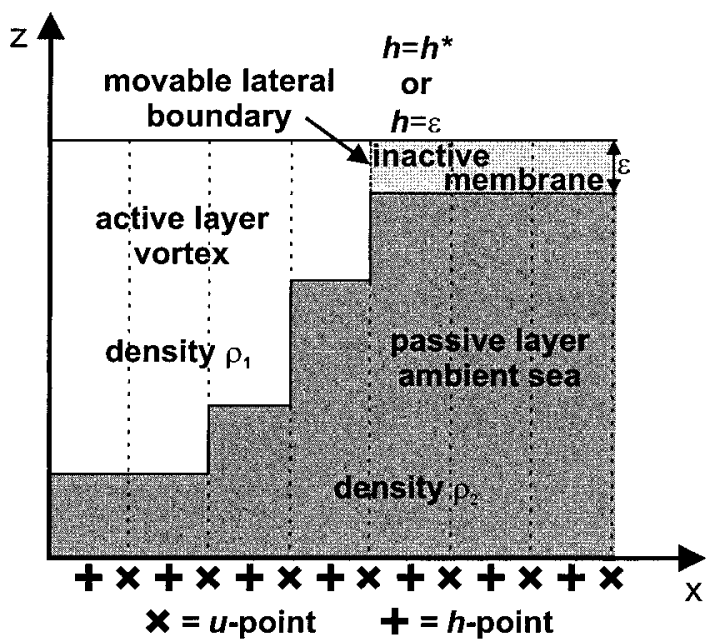

FIG. 1. Section through the discrete integration domain (Arakawa $\mathrm{C}$ grid) showing a schematic representation of the technique used in our model for the numerical treatment of movable lateral boundaries. Here $h^{*}$ denotes the provisional value calculated for the eddy thickness and $\varepsilon$ the critical layer thickness.

(e.g., Csanady 1979; Nof and Simon 1987; Pavia and Cushman-Roisin 1988; Rogers 1989; Olson 1991; Pavia and Lopez 1994; Matsuura 1995; Cushman-Roisin and Merchant-Both 1995; Rubino et al. 1998a; Ochoa et al. 1998; Esenkov and Cushman-Roisin 1999). One of the most commonly used theoretical models for the study of frontal, warm-core eddies consists in the nonlinear, reduced-gravity, shallow-water equations on an $f$ plane (Csanady 1979; Killworth 1983; Cushman-Roisin 1987; Pavia and Cushman-Roisin 1988; Pavia and Lopez 1994; Ochoa et al. 1998; Rubino et al. 1998a). As the degree of realism that can be achieved by using these equations is limited (they can, in fact, only describe the dynamics of an upper layer lying on the top of an infinitely deep, motionless ocean), they do not allow for important oceanic processes such as baroclinic instabilities, interactions between eddy and barotropic or baroclinic currents or features of the bottom topography or energy radiation via internal wave emission. Nevertheless, by solving these equations, many fundamental characteristics of the eddy dynamics can be studied. Moreover, they admit analytical, nonstationary solutions that describe the temporal and spatial evolution of a special class of frontal, warmcore eddies (Cushman-Roisin 1987; Rogers 1989; Rubino et al. 1998a). The fundamental representative of such a class is the pulson (Cushman-Roisin 1987). It is characterized by a paraboloidic shape and horizontal velocity components that are linear functions of the horizontal coordinates. This solution consists substantially of a pulsation of the eddy in which contractions and deepenings, expansions and shoalings, alternate during an exact inertial period.

Analytical solutions of nonlinear equations describing aspects of the dynamics of geophysical fluids are quite rare and, apart from their intrinsic value and fascination, they offer an ideal possibility to test quantitatively and systematically the accuracy of numerical models (Cushman-Roisin 1987; Sun et al. 1993). Such an opportunity is very valuable, especially in the case of numerical simulations of frontal, warm-core eddies, where one is faced with the treatment of a surface front. Its numerical description is, in fact, nontrivial as it requires the implementation of special algorithms allowing for the free horizontal eddy expansion and contraction. A continuously stratified model could be thus thought of as the most suitable tool for describing realistically the dynamics of localized water masses having an outcropping interface as well as their long-term behavior including their frictional and nonfrictional decay. However, an accurate description of the temporal and spatial evolution of a surface front requires an often unsustainably high computational effort as it requires a high horizontal and vertical resolution over large areas of the integration domain (Esenkov and Cushman-Roisin 1999). For this reason more efficient simulation methods have been proposed that are derived, substantially, from two different approaches to the problem of describing horizontal discontinuities in the water density. Pavia and Cushman-Roisin (1988, 1990) adapted the particle-in-cell method to oceanography: The frontal line can be traced here as the envelope of the particles contained in the surface layer. Using this method, Pavia and Cushman-Roisin were able to simulate, in a reduced-gravity context, the dynamics of a stationary anticyclonic circular frontal vortex and of an unstable elliptical frontal vortex, as well as the merging of anticyclonic frontal vortices; Pavia and Lopez (1994) studied the form variations in the evolution of elongated warm-core eddies in a reduced-gravity context using a least squares fitting procedure, while Esenkov and Cushman-Roisin (1999) extended these simulations to a two-active-layer system. However, no attempt was made, using this numerical technique, to quantify the eddy decay due to numerical energy dissipation as well as due to energy dissipation caused by frictional effects, nor was a test elucidating the capability of such a model of reproducing existing nonstationary analytical solutions that describe frontal vortices performed.

The second approach consists in the development of layered models equipped with numerical techniques for the treatment of movable lateral boundaries. Using these simulation techniques several oceanographic investigations were performed: Garvine (1982, 1984, 1987, 1996) developed different steady-state layered models for the simulation of frontal, buoyant plume dynamics in the frame of the reduced-gravity and of the two-active-layer approximation, while O'Donnell and Garvine (1983) and O'Donnell (1988, 1990) developed time-dependent models for the investigation of nonstationary aspects of similar frontal phenomena. Sun et al. (1993) reproduced qualitatively known steady-state analytical solutions describing wind- and buoyancy-forced flows characterized by the surfacing of a lower layer in a two- and three-layer ocean; Ju- 
ngclaus and Backhaus (1994) studied the intrusion of a bottom-arrested current in a quiescent deep ocean; Brandt et al. (1997) investigated the two-layer hydraulics of a narrow channel where the lower layer may intercept the bottom topography and compared numerical results with results obtained experimentally; Rubino et al. (1998b) studied the dynamics of underwater mudslides and the runup on a coast of the long surface waves that they generate. The results of these investigations encourage us to use a frontal, layered model for the description of the dynamics of surface frontal vortices, in particular of their frictional decay. A deep comprehension of the mechanisms responsible for the vortex decay (and even of the origin of the discrepancies often encountered between model results and observations) could, in fact, contribute to elucidate the role played by different physical processes in the observed long-term evolution of these coherent structures. To achieve this goal, a quantification of the model capability to reproduce the nonstationary dynamics of stable inviscid surface frontal vortices is essential. We thus compare, for the first time, numerical and analytical nonstationary solutions of the nonlinear, reduced-gravity, shallow-water equations for a horizontally homogeneous, inviscid, immiscible fluid describing stable frontal warm-core eddies quantitatively (note that such a comparison, on which the credibility of the results provided by a numerical model for the description of frontal vortices crucially depends, has never been performed before, even qualitatively). As a result, a quantification of the numerical dissipation affecting the simulated solutions is obtained. On this basis, the eddy decay due to three different mechanisms, namely interfacial friction, horizontal momentum diffusion, as well as ambient-water entrainment, is investigated. Finally, a critical discussion about the appropriateness of the different approximations assumed in our investigation is presented. In particular, the appropriateness of the reduced-gravity assumption for the description of warm-core eddies is tested by estimating qualitatively the differences between the evolution of a warm-core eddy in a two-active-layer ocean of different depths.

The paper is organized as follows: In section 2 the numerical model is presented and aspects of the numerical techniques implemented are discussed. In section 3 numerical simulations for the case of a horizontally homogeneous, inviscid, immiscible fluid are carried out, their results are compared with the analytical nonstationary (pulson) solution, and the numerical dissipation affecting the simulated solutions is quantified. The influence of interfacial friction, horizontal momentum diffusion, as well as ambient water entrainment on eddy decay is investigated in section 4 . In section 5 the appropriateness of the reduced-gravity assumption is discussed. Finally, in section 6 the results of the investigations are discussed critically and conclusions are presented.

\section{The numerical model}

\section{a. Model equations in the frame of the reduced- gravity approximation}

The equations that constitute our model in the frame of the reduced-gravity approximation are the nonlinear, hydrostatic, shallow-water equations for a horizontally inhomogeneous, viscous fluid on an $f$ plane. They include terms describing horizontal momentum diffusion as well as vertical shear stress and ambient-water entrainment at the interface between the active surface layer and the quiescent environment. In the following we denote the surface layer thickness by $h$ and the vertically averaged velocity and transport vectors within this layer by $\mathbf{u}$ and $\mathbf{U}=\mathbf{u} h$. Then, the momentum equation, the continuity equation, and the evolution equation for the water density read:

$$
\begin{gathered}
\frac{\partial \mathbf{U}}{\partial t}+\boldsymbol{\nabla}_{h} \cdot(\mathbf{u} \times \mathbf{U})+\mathbf{F}_{h} \cdot \mathbf{U} \\
=-g^{\prime} h \boldsymbol{\nabla}_{h} h-\frac{g h^{2}}{2 \bar{\rho}} \nabla_{h} \rho-\frac{\tau_{i}}{\bar{\rho}}+A_{h} \nabla_{h}^{2} \mathbf{U}-\omega \mathbf{u}, \\
\frac{\partial h}{\partial t}+\nabla_{h} \cdot \mathbf{U}=\omega, \quad \text { and } \\
\frac{\partial \rho}{\partial t}+\mathbf{u} \cdot \nabla_{h} \rho+\omega \frac{\rho-\rho_{a}}{h}=0 .
\end{gathered}
$$

Here $\boldsymbol{\nabla}_{h}$ denotes the horizontal Nabla operator, - the scalar product, and $\times$ the tensor product. The two-dimensional Coriolis matrix $\mathbf{F}_{h}$ is defined as

$$
\mathbf{F}_{h}=\left(\begin{array}{cc}
0 & -f \\
f & 0
\end{array}\right),
$$

where $f$ is the Coriolis parameter. The water densities in the surface layer and in the ambient ocean are $\rho$ and $\rho_{a}$ respectively, $\bar{\rho}=\left(\rho+\rho_{a}\right) / 2$ is the mean water density, $g^{\prime}=g\left(\rho_{a}-\rho\right) / \bar{\rho}$ is the reduced gravity, where $g$ denotes the acceleration of gravity, and $A_{h}$ the horizontal eddy diffusion coefficient. In our investigation the vertical shear stress at the interface $\tau_{i}$ will be assumed to depend on the horizontal current velocity within the surface layer linearly:

$$
\frac{\tau_{i}}{\bar{\rho}}=c_{\mathrm{dil}} \mathbf{U},
$$

or quadratically:

$$
\frac{\tau_{i}}{\bar{\rho}}=c_{\mathrm{dil}} \mathbf{u}|\mathbf{u}| .
$$

In the previous formulas $c_{\mathrm{dil}}$ and $c_{\mathrm{diq}}$ denote the drag coefficients at the interface. The entrainment velocity $\omega$ will be assumed to be proportional to the horizontal current velocity within the surface layer (Csanady 1979):

$$
\omega=\lambda|\mathbf{u}|,
$$


where $\lambda$ denotes the nondimensional entrainment coefficient. Note that this parameterization allows only for one-way entrainment, which transfers water from the quiescient ambient fluid into the active layer. In the following the ambient water density $\rho_{a}$ as well as the coefficients $A_{h}, c_{\mathrm{dil}}, c_{\mathrm{diq}}$, and $\lambda$ will be assumed to be constant in our model.

\section{b. Aspects of the used numerical techniques}

Equations (1)-(3) are discretized on a staggered Arakawa $\mathrm{C}$ grid. The numerical scheme adopted for their integration uses forward differences in time and central differences in space. The advective terms are solved by means of a directional upstream algorithm (Jungclaus and Backhaus 1994; Rubino et al. 1998b). Consider the discrete expression of the temporal derivative:

$$
\frac{\partial \mathbf{U}}{\partial t}=\frac{\mathbf{U}^{n+1}-\mathbf{U}^{n}}{\Delta t}
$$

where $\mathbf{U}^{n+1}$ represents the transport at the time $t^{n+1}=$ $(n+1) \Delta t, \mathbf{U}^{n}$ the transport at $t^{n}=n \Delta t$, and $\Delta t$ the temporal increment. The momentum equation can be discretized as follows:

$$
\mathbf{U}^{n+1}=\mathbf{M}_{1} \mathbf{U}^{n}-\mathbf{M}_{2}\left(\hat{\boldsymbol{\nabla}}_{h} \cdot\left(\mathbf{u}^{n} \times \mathbf{U}^{n}\right)-g^{\prime} h \hat{\boldsymbol{\nabla}}_{h} h+\frac{g h^{2}}{2 \bar{\rho}} \hat{\boldsymbol{\nabla}}_{h} \rho\right)+\Delta t\left(-\frac{\tau_{i}}{\bar{\rho}}+A_{h} \hat{\nabla}_{h}^{2} \mathbf{U}^{n}-\omega \mathbf{u}^{n}\right),
$$

with

$$
\mathbf{M}_{1}=\left[\begin{array}{cc}
\cos (f \Delta t) & \sin (f \Delta t) \\
-\sin (f \Delta t) & \cos (f \Delta t)
\end{array}\right] \text { and } \quad \mathbf{M}_{2}=\frac{1}{f}\left[\begin{array}{cc}
\sin (f \Delta t) & 1-\cos (f \Delta t) \\
-(1-\cos (f \Delta t)) & \sin (f \Delta t)
\end{array}\right]
$$

Note that in (9) $\hat{\nabla}_{h}$ is the discrete form of the horizontal Nabla operator. Solving (9) has the advantage that, in the inviscid case, analytical stationary currents can be reproduced very accurately (Backhaus 1985; Burchard 1995; Hessner 1998). This is a very valuable property of our numerical scheme, as it reduces strongly the energy decay induced by numerical inaccuracies in flow regimes dominated by a balance between Coriolis force, pressure gradient, and advection.

The model is a frontal model. In fact, in this model a special technique for the numerical treatment of movable lateral boundaries allows for the description of the temporal and spatial evolution of a localized layer with an outcropping interface on the top of a dynamically passive environment. It is possible to treat the domain of integration as composed of one active and one passive layer for those points where the water column is vertically stratified (i.e., where the vortex exists) and of only one passive layer and a thin membrane (its thickness, $\varepsilon$, is typically a few centimeters), dynamically inactive, elsewhere. Referring to Fig. 1, which shows a section through the discrete integration domain, the technique for determining the variable position of the lateral boundaries (i.e., for tracing the vortex rim) can be elucidated as follows.

As known, in the Arakawa C grid a "transport" point (i.e., a point where transport values are calculated) lies between two "thickness" points (i.e., points where layer thicknesses are calculated). At every time step, a provisional transport value is calculated only in those transport points for which at least the value of one of the two thicknesses in the neighboring thickness points is larger than the critical value $\varepsilon$ (obviously, the transport values in the remaining transport points are and remain zero). By solving the continuity equation (2), provisional values for the layer thicknesses are obtained. Due to unavoidable numerical inaccuracies, some of these values referring to thickness points located near the vortex rim will be negative. This "virtual water" (Backhaus 1976) can be eliminated by introducing a corrective algorithm that, acting on the transport points neighboring a negative thickness point and eventually iteratively on the transport points in the whole integration domain, reduces negative thicknesses to zero. The corrected transport and thickness values are used to calculate the solution at the next time step. Similar techniques for the treatment of movable lateral boundaries at the sea bottom were implemented, for example, by Jungclaus and Backhaus (1994) and Rubino et al. (1998b).

\section{Comparison between numerical and analytical solutions describing nonstationary, frontal, warm-core eddies}

In order to test the capability of our numerical model of simulating the dynamics of stable frontal warm-core eddies, we carried out, for the first time, numerical simulations aimed at reproducing the spatial and temporal evolution of a pulson, which represents an analytical, nonstationary solution of the nonlinear, reduced-gravity, shallow-water equations for a homogeneous, inviscid, immiscible fluid on an $f$ plane (Cushman-Roisin 1987). As we noted in our introduction, this step seems to be a necessary one, as far as the numerical simulation of 
different phenomena related to the dynamics of frontal surface vortices is concerned. In fact, the credibility and utility of numerical primitive equation layered models in the description of frontal phenomena in general, and of frontal surface vortices in particular, depends significantly on their ability in reproducing isopycnic layer outcropping at the sea surface. In this frame, the reproduction of analytical, nonstationary solutions of the nonlinear shallow-water equation represents an unique opportunity (Sun et al. 1993). The pulson is a class of nonlinear, oscillating, frontal anticyclonic surface eddies whose thickness $h$ has the form of a circular paraboloid and whose horizontal velocity components $u$ and $v$ are linear functions of the horizontal coordinates $x$ and $y$ :

$h=\alpha H_{0}-\alpha^{2} \frac{H_{0}}{R_{0}^{2}}\left(x^{2}+y^{2}\right)$,

$h=\frac{1}{2} \alpha \beta f x+\frac{f}{2}\left(1-\alpha \sqrt{1-\gamma^{2}-\frac{8 g^{\prime} H_{0}}{f^{2} R_{0}^{2}}}\right) y$,

$v=-\frac{f}{2}\left(1-\alpha \sqrt{1-\gamma^{2}-\frac{8 g^{\prime} H_{0}}{f^{2} R_{0}^{2}}}\right) x+\frac{1}{2} \alpha \beta f y$,

where

$$
\alpha=\frac{1}{1+\gamma \sin (f t+\varphi)} \quad \text { and } \quad \beta=\gamma \cos (f t+\varphi) .
$$

Here $H_{0}$ represents the mean thickness of the pulson at its center (which, in our notation, coincides with the origin of the coordinate system), $R_{0}$ its mean radius at the sea surface, and $\gamma$ and $\varphi$ the strength and phase of the oscillations that characterize the pulson evolution. The central experiment of our model test (to this experiment we will often refer in the following) was performed for a pulson characterized by $H_{0}=400 \mathrm{~m}, R_{0}$ $=120 \mathrm{~km}, \gamma=0.1, f=7 \times 10^{-5} \mathrm{~s}^{-1}, g^{\prime}=9.81 \times$ $10^{-3} \mathrm{~m} \mathrm{~s}^{-2}, A_{h}=0, c_{\mathrm{dil}}=0, c_{\mathrm{diq}}=0, \lambda=0$, and $\varphi$ $=-\pi / 2$ for $t=0$. The horizontal grid steps $\Delta x$ and $\Delta y$ and the time step $\Delta t$ were chosen to be $1200 \mathrm{~m}$ and $360 \mathrm{~s}$. The numerical simulation was carried out over 15 inertial periods $T=2 \pi / f$ as, for a pulson characterized by the above mentioned parameters, this time is of the same order as the time needed for nonstationary frontal processes to develop in the frame of the frontal geostrophic dynamics (Cushman-Roisin 1986; Pavia and Cushman-Roisin 1988).

Figure 2 shows the normalized thickness (i.e., the thickness divided by $H_{0}$ ) as well as the normalized horizontal velocity field (i.e., the velocity divided by $f R_{0}$ ) as a function of the normalized coordinates (i.e., $x / R_{0}$ and $y / R_{0}$ ) of the simulated pulson for four selected times within an inertial period. In this figure the upper panels give the vertical sections of the simulated pulson thickness through its center as well as the corresponding analytical vertical sections for the time $t=0$ (time of maximum pulson center depth) and for the time $t=(1 / 2) T$ (time of minimum pulson center depth). In the lower panels of Fig. 2 the horizontal velocity field as well as selected isolines of the simulated pulson thickness are presented. Figure 2a shows the initial conditions for the simulated pulson [Eqs. (11)-(13) with the aforementioned pulson parameters]. For $t=0$ the pulson has reached its maximum center depth, while the corresponding horizontal velocity field is solenoidal and, in each point, the absolute value of the horizontal velocity vector is minimum. At $t=(1 / 4) T$, the simulated pulson is shoaling and expanding, while the corresponding horizontal velocity field is now divergent. At $t=(1 / 2) T$ the pulson has reached its minimum center depth and its maximum radius, while the corresponding horizontal velocity field is again solenoidal and, in each point, the absolute value of the horizontal velocity vector is maximum. A comparison between analytical and numerical pulson thicknesses (Fig. 2c, upper panel) reveals that only marginal deviations near the pulson rim exist. At $t=(3 / 4) T$, the simulated pulson is deepening and contracting, while the corresponding horizontal velocity field is now convergent.

In order to illustrate the long-term evolution of the simulated pulson we compared the temporal evolution of the analytical pulson radius $R$ at the sea surface:

$$
R=\frac{R_{0}}{\sqrt{\alpha}},
$$

of the analytical pulson center depth $H$ :

$$
H=\alpha H_{0},
$$

of the analytical potential energy density $E_{p}$ :

$$
E_{p}=\frac{1}{3} g^{\prime} \alpha H_{0} .
$$

of the analytical kinetic energy density $E_{k}$ :

$$
E_{k}=\frac{1}{12} R_{0}^{2} f^{2}\left(1-\sqrt{1-\gamma^{2}-\frac{8 g^{\prime} H_{0}}{f^{2} R_{0}^{2}}}-\alpha \frac{4 g^{\prime} H_{0}}{f^{2} R_{0}^{2}}\right),
$$

and of the analytical total energy density $E=E_{p}+E_{k}$ :

$$
E=\frac{1}{12} R_{0}^{2} f^{2}\left(1-\sqrt{1-\gamma^{2}-\frac{8 g^{\prime} H_{0}}{f^{2} R_{0}^{2}}}\right)
$$

with the temporal evolution of the corresponding quantities as obtained by using our numerical model. Figure 3 shows the temporal evolution of the normalized pulson thickness at its center (Fig. 3a), of the normalized pulson radius at the sea surface (Fig. 3b), and of the normalized pulson potential, kinetic, and total energy densities (Fig. 3c) as calculated analytically and as calculated by using our numerical model. The simulation results show that our numerical model is able to simulate with a very good accuracy the main characteristics of the pulson evolution. From the time series of pulson radius, center depth, as well as potential and kinetic energy density, the presence of inertial oscillations is evident. In par- 

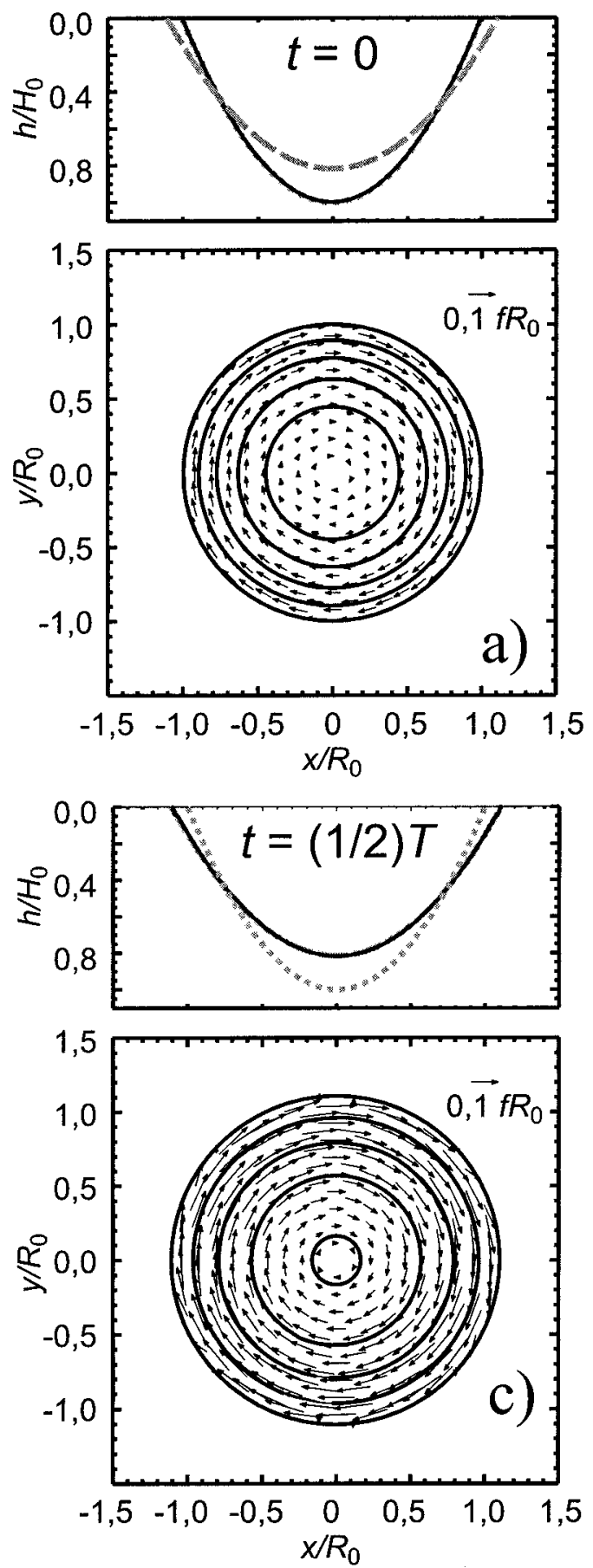
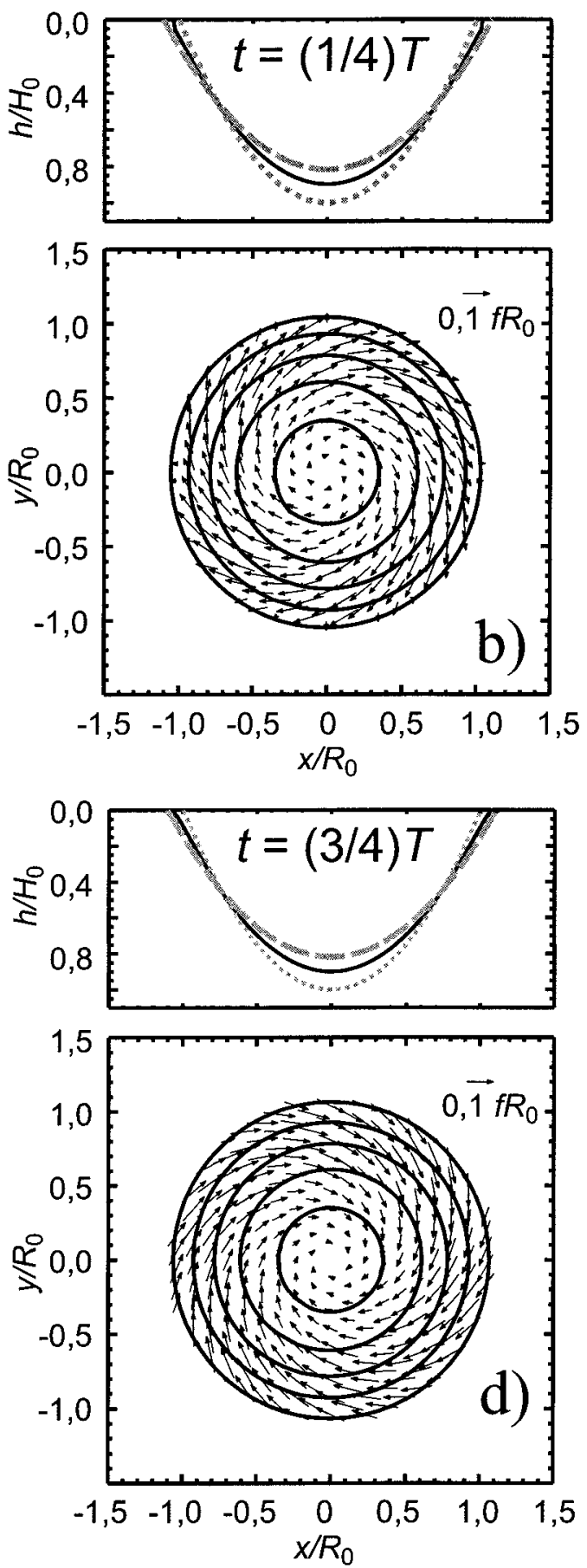

FIG. 2. Normalized thickness (upper panels) and normalized horizontal velocity field (lower panels) of the simulated pulson for four selected times within an inertial period $T$. In the upper panels the normalized thicknesses of the analytical pulson for the time $t=0$ (dotted gray lines) and for the time $t=(1 / 2) T$ (dashed gray lines) are also shown. In the lower panels selected isolines of the simulated pulson thickness are also presented.

ticular, potential and kinetic energy density pulsate with the same amplitude and are exactly in phase opposition. Also a predicted asymmetry of the analytical pulson solution is captured by our numerical model: The period spent by the pulson in its shallow-wide state is larger than the period spent by the pulson in its deep-narrow state.

A comparison between the spectral analyses of the time series of the pulson center depth as obtained analytically and as calculated by using our numerical mod- 


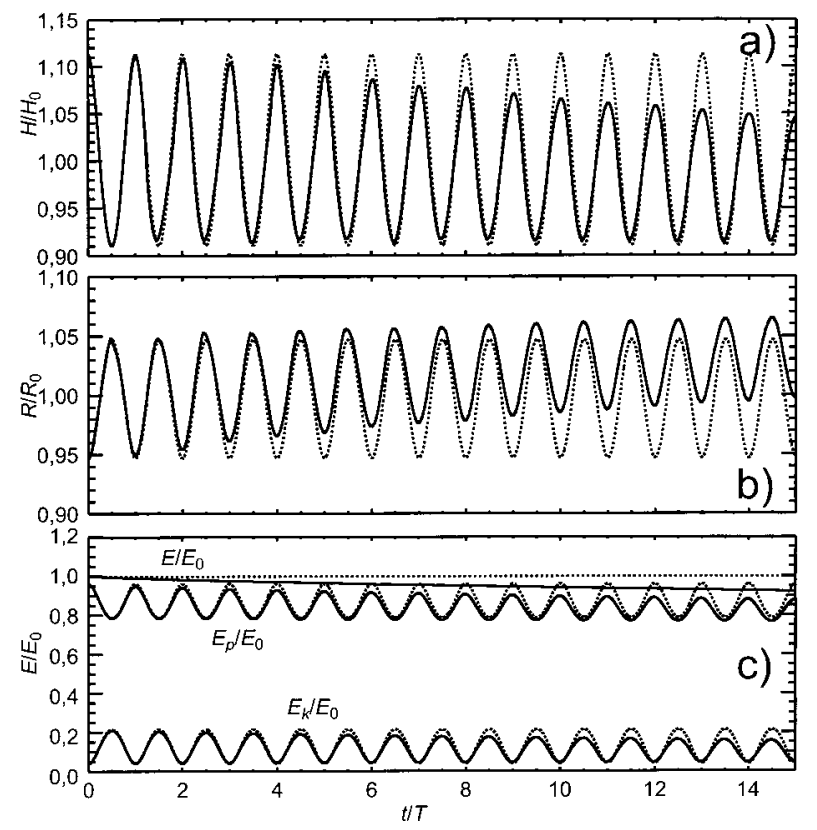

FIG. 3. Time series of the normalized pulson thickness at its center (a) of the normalized pulson radius at the sea surface (b), and of the normalized pulson potential, kinetic, and total energy densities $E_{p}$, $E_{k}$, and $E$ (c) for the central experiment (see text). Solid lines denote the results of our numerical simulation, dotted lines are obtained analytically. Note that $E_{0}$ represents the pulson total energy density.

el is presented in Fig. 4. Our model reproduces quite well frequency and amplitude of the fundamental as well as of the second harmonic characterizing the analytical pulson oscillation and it also captures the peak corresponding to the third one.

A deviation between analytical and numerical solution can be noted in each of the curves presented in Fig. 3. However, after 15 inertial periods more than $92 \%$ of the initial total energy density is conserved by our numerical model. Assuming an exponential decay of the total energy density associated with the simulated pulson, we may derive an $e$-folding time, $\hat{T}$, for the total energy density of the simulated pulson. For the present simulation it results $\hat{T} \cong 192 \mathrm{~d}$, that is, after 192 days the pulson is still a very robust feature, its energy being $1 / e$ of its initial value. This implies that the lifetime of our numerical pulson is larger than the lifetime of most of the surface frontal eddies observed in the World Ocean (Csanady 1979; Olson 1991).

In Fig. 5 the normalized energy dissipation rate as well as the normalized $e$-folding time of different simulated pulsons as a function of the pulson mean radius $R_{n}$ relative to $R_{0}$ and as a function of the pulson oscillation strength is presented. The remaining model parameters are the same used for the central experiment discussed above. The lifetime of the simulated pulsons increases with pulson mean radius. In general, larger oscillations yield shorter lifetimes. However, this dependence decreases with decreasing pulson mean radius.

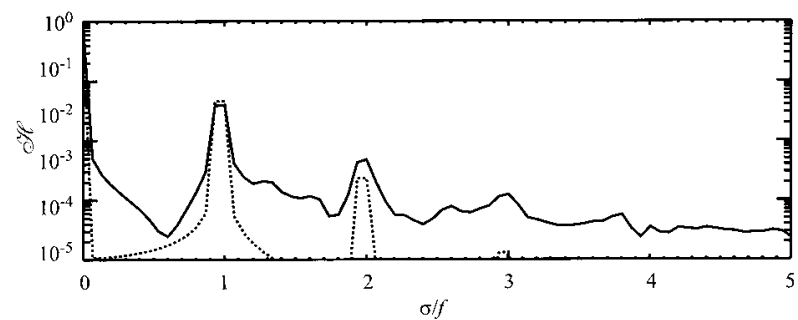

FIG. 4. Spectra of the discrete time series of the pulson center depth as obtained analytically (dotted line) and as calculated by using our numerical model in the central experiment (solid line).

Note that the finite values of the lifetimes of the simulated pulsons presented above result uniquely from the numerical dissipation inherent in the discrete nature of our numerical model. These values are large enough to permit a quantitative discussion on the role played by different physical mechanisms in the decay of simulated pulsons.

\section{Frictional decay of frontal, warm-core eddies}

The decay of frontal warm-core eddies can derive from different phenomena related to their interactions with the atmosphere (heat exchange, wind stress input) with the interior ocean (friction, diabatic processes, wave emission) and with topographic features (impact with coasts or bottom topography). But, it can also be a consequence of instability processes leading to the vortex disintegration (Stegner and Dritschel 2000).

In order to elucidate aspects of the frictional decay of stable frontal warm-core eddies, we discuss, in the present section, how three different mechanisms affect their temporal and spatial evolution. The three mechanisms considered in our investigation are interfacial (linear as well as quadratic) friction, harmonic horizontal momentum diffusion, as well as linear ambient-water entrainment. The parameterizations used for describing these mechanisms (see section 2) are among the most common ones applied in ocean modeling. A critical discussion about the appropriateness of these parameterizations will be presented in the final section. The initial

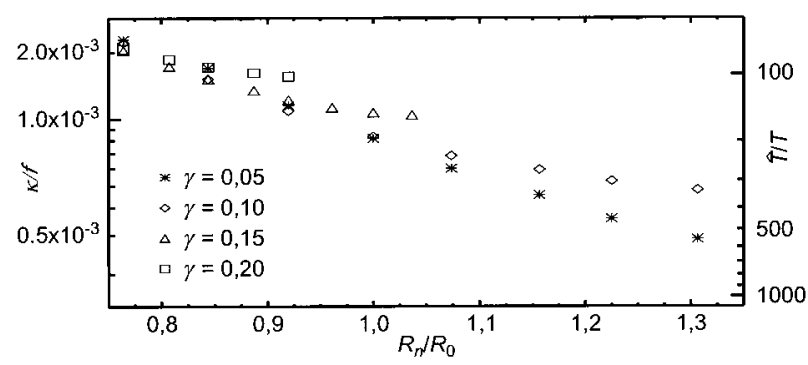

FIG. 5. Normalized energy dissipation rate and normalized $e$-folding time of different simulated pulsons as a function of the pulson mean radius $R_{n}$ relative to the pulson mean radius $R_{0}$ of our central experiment (see text) and as a function of the pulson oscillation strength $\gamma$. 


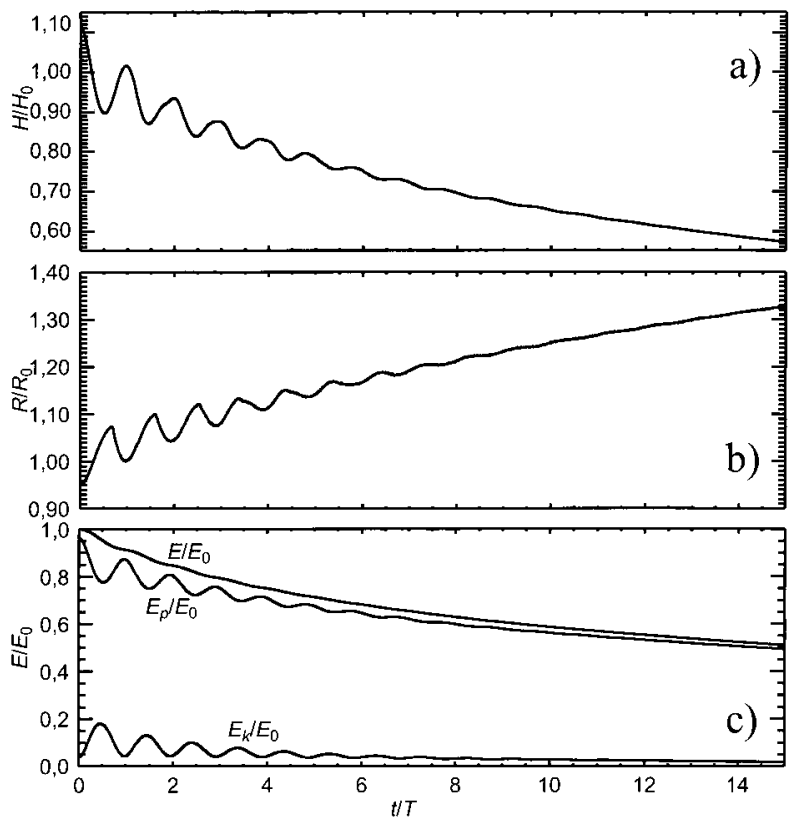

FIG. 6. Time series of the normalized pulson thickness at its center (a), of the normalized pulson radius at the sea surface (b), and of the normalized pulson potential, kinetic, and total energy densities $E_{p}$, $E_{k}$, and $E(\mathrm{c})$ for the experiment including linear interfacial friction.

conditions imposed in the following simulations are the same chosen for the central experiment discussed in the previous section.

\section{a. Interfacial friction}

\section{1) LINEAR PARAMETERIZATION}

Figure 6 shows the temporal evolution of the normalized vortex thickness at its center (Fig. 6a), of the normalized vortex radius at the sea surface (Fig. 6b), and of the normalized vortex potential, kinetic, and total energy densities (Fig. 6c) as calculated by using our numerical model for a vortex evolving under the influence of an interfacial friction obeying a linear drag law [see Eq. (5)]. The aperiodicity induced in the vortex evolution by the use of a value of the drag coefficient $\left(c_{\text {dil }}=4 \times 10^{-6} \mathrm{~s}^{-1}\right)$, which belongs to the range of values commonly used in ocean modeling of the nearsurface layer (Neumann and Pierson 1966), is remarkable. In particular the inertial pulsations are substantially attenuated. The vortex flattens and shoals as the system energy rapidly dissipates. Note, however, that this dissipation drops off rapidly once the motion slows. The $e$-folding time for energy dissipation is in this experiment $\hat{T} \cong 22 \mathrm{~d}$, which corresponds approximately to a nine-time-faster decay compared to the decay obtained in the central experiment. In Fig. 7 the $e$-folding time for energy decay caused by linear interfacial friction is illustrated for 12 different experiments carried out by varying the value of the drag coefficient. For realistic values of the linear friction coefficient we obtain $e$-fold-

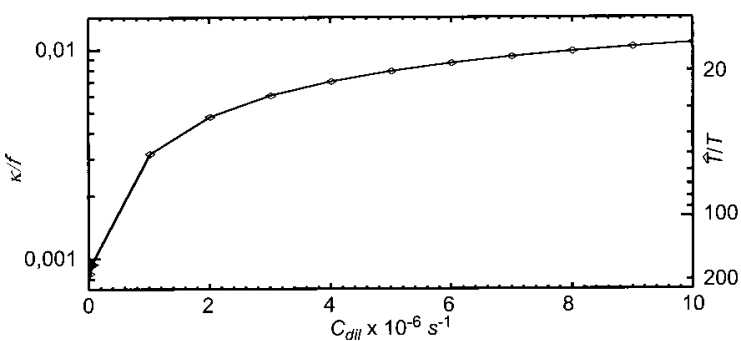

FIG. 7. Dissipation rate and $e$-folding time for energy decay due to linear interfacial friction as a function of the drag coefficient. The black diamond gives the value obtained using $c_{\mathrm{dil}}=3.2 \times 10^{-8} \mathrm{~s}^{-1}$, which corresponds to the value used by Matsuura (1980).

ing times in agreement with the result obtained by Csanady (1979) (who, however, used a quadratic parameterization, see next point), although the vortex pulsation was neglected in his investigation. We carried out also a simulation using for the linear friction coefficient the very small value $\left(c_{\mathrm{dil}}=3.2 \times 10^{-8} \mathrm{~s}^{-1}\right)$ used by Matsuura (1980) for the simulation of the decay of strong cyclonic and anticyclonic vortices. The energy dissipation obtained in this case is virtually identical to that obtained in the inviscid case.

\section{2) QUADRATIC PARAMETERIZATION}

Figure 8 shows the temporal evolution of the normalized vortex thickness at its center (Fig. 8a), of the normalized vortex radius at the sea surface (Fig. 8b), and of the normalized vortex potential, kinetic, and total energy densities (Fig. 8c) as calculated by using our numerical model for a vortex evolving under the influence of an interfacial friction obeying a quadratic drag law [see Eq. (6)]. Like in the previous case, the aperiodicity induced in the vortex evolution by the use of a value of the drag coefficient $\left(c_{\text {diq }}=3 \times 10^{-4}\right)$ belonging to the range of values commonly used in ocean modeling for sharp and stable interfaces (Csanady 1978, 1979 ) is remarkable. The inertial pulsations are very strongly attenuated, their presence being hardly recognizable after 5 inertial periods. Also in this case, the energy dissipation drops off rapidly once the motion slows. Like in the previous case, the $e$-folding time for energy dissipation is in this experiment $\hat{T} \cong 22 \mathrm{~d}$. In Fig. 9 the $e$-folding time for energy decay due to quadratic interfacial friction is illustrated for 11 different experiments carried out by varying the value of the drag coefficient. Also in this case, for realistic values of the drag coefficients we obtain $e$-folding times in agreement with the result obtained by Csanady (1979). A comparison between the results obtained using linear and quadratic interfacial friction that yield approximately the same $e$-folding time (cf. Figs. 7a and 8a and Figs. $7 \mathrm{~b}$ and $8 \mathrm{~b}$ ) shows that quadratic friction induces a smaller decrease in the vortex thickness at its center and a larger increase in the vortex radius than linear friction. This is due to the fact that linear friction is more efficient 


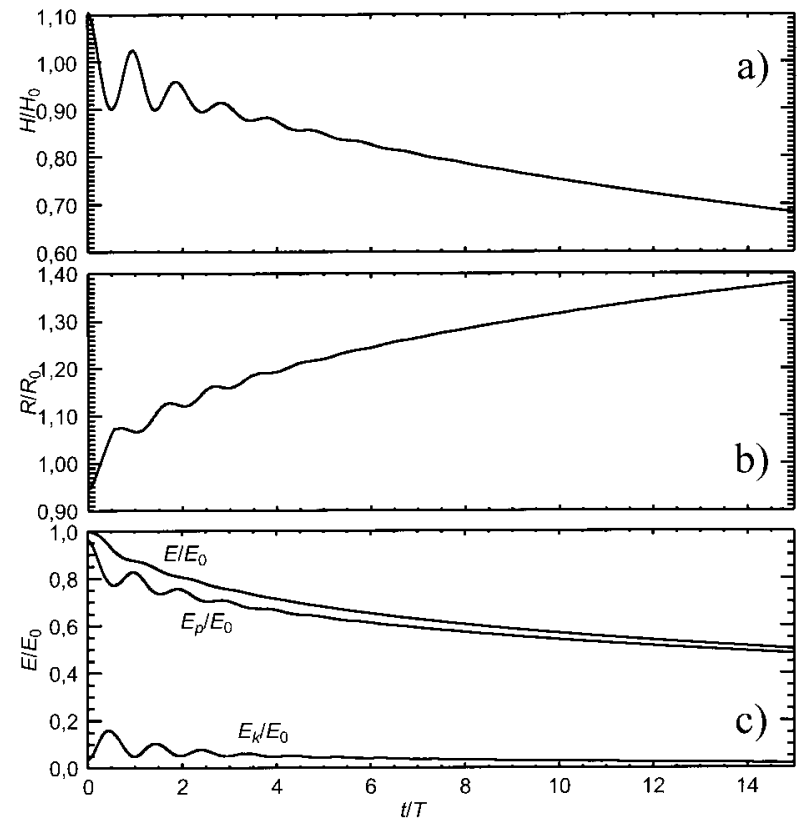

FIG. 8. Time series of the normalized pulson thickness at its center (a), of the normalized pulson radius at the sea surface (b), and of the normalized pulson potential, kinetic, and total energy densities $E_{p}$, $E_{k}$, and $E$ (c) for the experiment including quadratic interfacial friction.

than quadratic friction near the vortex axis, while the opposite is true near the vortex periphery. Moreover it appears that quadratic friction is more efficient than linear friction in damping the vortex pulsation. However, for realistic values of the drag coefficients, both parameterizations induce a strong energy decay.

\section{b. Horizontal momentum diffusion}

Figure 10 shows the temporal evolution of the normalized vortex thickness at its center (Fig. 10a), of the normalized vortex radius at the sea surface (Fig. 10b), and of the normalized vortex potential, kinetic, and total energy densities (Fig. 10c) as calculated by using our numerical model for a vortex evolving under the influence of a horizontal momentum diffusion obeying a harmonic law [see Eq. (1)]. For a vortex following exactly the pulson dynamics such kind of dissipation mechanism would be ineffective, as the pulson velocity components are linear functions of the horizontal coordinates. The aperiodicity induced in the vortex dynamics by including harmonic horizontal momentum diffusion in the physics governing the vortex evolution can thus be considered as a measure of the distance between analytical and numerical solution. By using a value for the horizontal eddy diffusion coefficient $\left(A_{h}\right.$ $=10 \mathrm{~m}^{2} \mathrm{~s}^{-1}$ ), which seems to be a reasonable value, given the used spatial resolution, only slight differences between the time series shown in Fig. 10 and the cor-

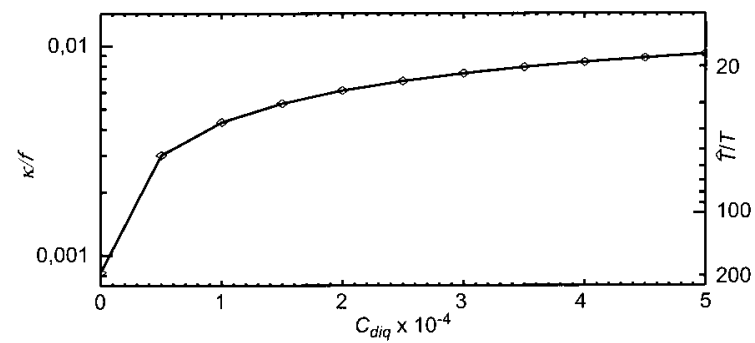

FIG. 9. Dissipation rate and $e$-folding time for energy decay due to quadratic interfacial friction as a function of the drag coefficient.

responding time series obtained in the central experiment (see Fig. 3) can be observed. The $e$-folding time for energy dissipation is in this experiment $\hat{T} \cong 144 \mathrm{~d}$, which is not much smaller than the corresponding $e$ folding time obtained in the central experiment. In Fig. 11 the $e$-folding time of the energy decay for 11 different experiments carried out by varying the value of the horizontal eddy diffusion coefficient is illustrated. From this figure it can be noted that the vortex decay is not particularly sensitive to variations in the horizontal eddy diffusion coefficient.

\section{c. "Ambient-water" entrainment}

Figure 12 shows the temporal evolution of the normalized vortex thickness at its center (Fig. 12a), of the

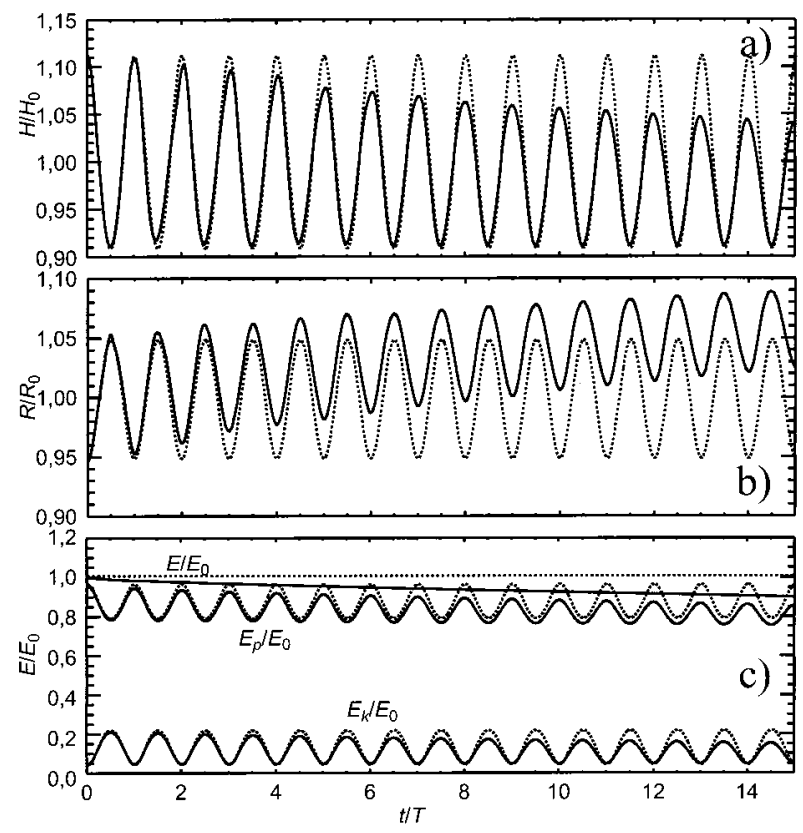

FIG. 10. Time series of the normalized pulson thickness at its center (a), of the normalized pulson radius at the sea surface (b), and (c) of the normalized pulson potential, kinetic, and total energy densities $E_{p}, E_{k}$, and $E$ (c) for the experiment including harmonic horizontal momentum diffusion. Solid lines denote the results of our numerical simulation, dotted lines are obtained analytically (pulson solution). 


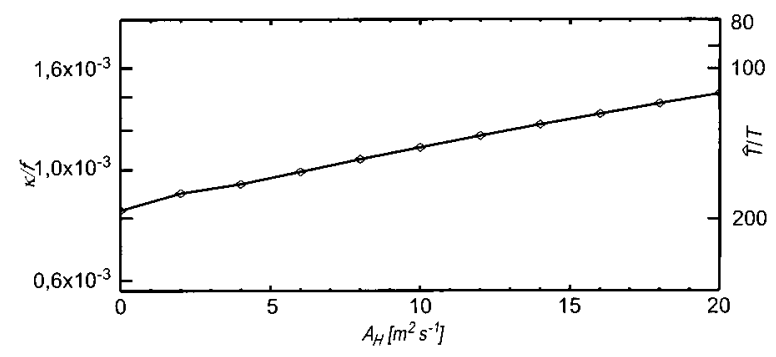

FIG. 11. Dissipation rate and $e$-folding time for energy decay due to harmonichorizontal momentum diffusion as a function of the horizontal eddy diffusion coefficient.

normalized vortex radius at the sea surface (Fig. 12b), and of the normalized vortex potential, kinetic, and total energy densities (Fig. 12c) as calculated by using our numerical model for a vortex that, during its evolution, entrains ambient water at its interface to the quiescent ocean through a vertical velocity depending linearly on the absolute value of the local horizontal velocity vector [see Eq. (7)] (Csanady 1979). The effect of ambientwater entrainment on the vortex dynamics is subtle, as it possesses a frictional component [see Eq. (1)] and a nonfrictional component. It increases the vortex volume [see Eq. (2)] and causes a decrease in the density contrast between surface layer and ambient ocean as well as horizontal inhomogeneities in the vortex density field [see Eq. (3)], thus contributing to the pressure distribution within the vortex [see Eq. (1)]. Csanady (1979), using an analytical reduced-gravity model, assessed the dissipation of the vortex available potential energy due to an entrainment parameterization similar to that used in our investigation but resulting in a mere mass transfer across the pycnocline that separates the active surface layer from the quiescent ocean. He found that, for large density gradients like those observed at the boundaries between warm core rings and ambient water, the vortex decay due to its volume gain induced by ambient-water entrainment is negligible compared to the vortex decay due to the energy dissipation induced by interfacial friction. These results are confirmed by our calculations. By using a value for the entrainment coefficient $\lambda=3$ $\times 10^{-7}$, which belongs to the range of values commonly used in ocean modeling (Csanady 1979), no major differences between the time series shown in Fig. 12 and the corresponding time series obtained in the central experiment (see Fig. 3) can be observed. The $e$-folding time for energy dissipation is in this experiment $\hat{T} \cong$ $181 \mathrm{~d}$, which is only slightly smaller than the corresponding $e$-folding time obtained in the central experiment. In Fig. 13 the $e$-folding time of the energy decay for 11 different experiments carried out by varying the value of the entrainment coefficient is illustrated. From this figure it can be noted that the vortex decay is not significantly affected by variations in the entrainment coefficient.

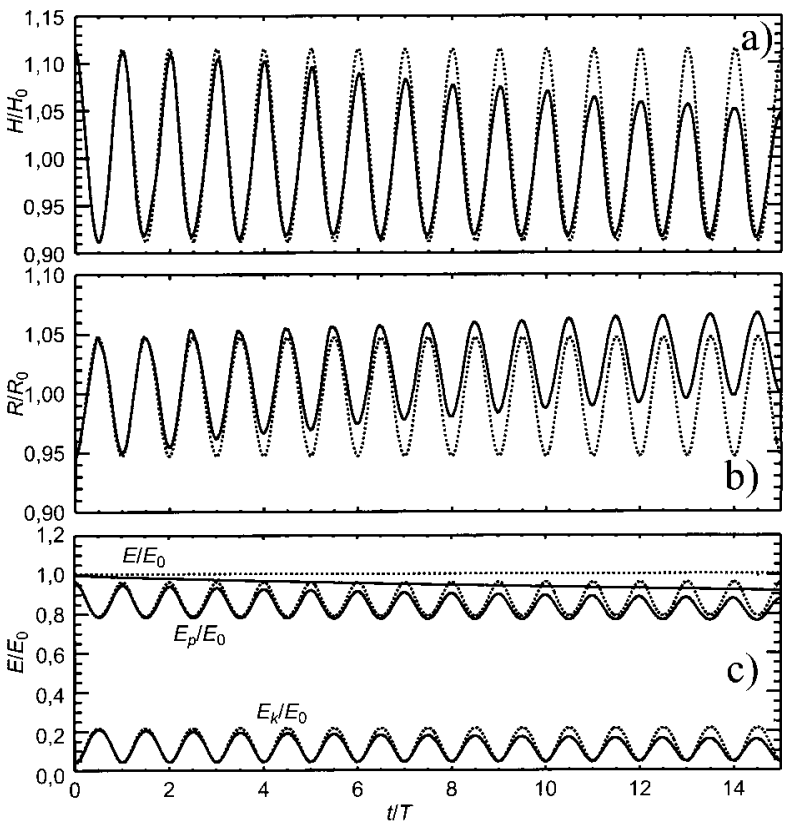

FIG. 12. Time series of the normalized pulson thickness at its center (a), of the normalized pulson radius at the sea surface (b), and of the normalized pulson potential, kinetic, and total energy densities $E_{p}$, $E_{k}$, and $E$ (c) for the experiment including linear ambient-water entrainment. Solid lines denote the results of our numerical simulation, dotted lines are obtained analytically (pulson solution).

\section{On the appropriateness of the reduced-gravity assumption in the simulation of warm-core eddies}

The appropriateness of the reduced-gravity assumption in the simulation of warm-core eddies will be discussed here, for the specific case object of our investigation, using results of an inviscid, immiscible twoactive-layer extension of our reduced-gravity model presented in section 2 . The equations, which constitute the two-layer model, are thusfor the upper layer:

$$
\begin{gathered}
\frac{\partial \mathbf{U}_{1}}{\partial t}+\boldsymbol{\nabla}_{h} \cdot\left(\mathbf{u}_{1} \times \mathbf{U}_{1}\right)+\mathbf{F}_{h} \cdot \mathbf{U}_{1}=-g h_{1} \boldsymbol{\nabla}_{h} \eta_{1}, \\
\frac{\partial \eta_{1}}{\partial t}+\nabla_{h} \cdot \mathbf{U}_{1}+\nabla_{h} \cdot \mathbf{U}_{2}=0
\end{gathered}
$$

and for the lower layer:

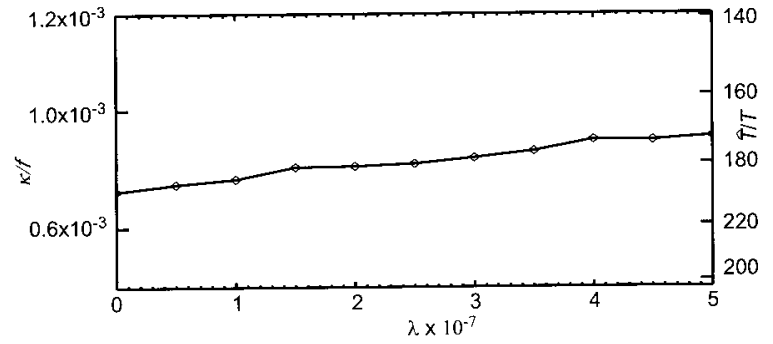

FIG. 13. Dissipation rate and $e$-folding time for energy decay due to linear ambient-water entrainment as a function of the entrainment coefficient. 


$$
\begin{aligned}
\frac{\partial \mathbf{U}_{2}}{\partial t}+\boldsymbol{\nabla}_{h} \cdot\left(\mathbf{u}_{2} \times \mathbf{U}_{2}\right)+\mathbf{F}_{h} \cdot \mathbf{U}_{2}= & -g h_{2} \boldsymbol{\nabla}_{h} \eta_{1} \\
& -g^{\prime} h_{2} \boldsymbol{\nabla}_{h} \eta_{2}, \\
\frac{\partial \eta_{2}}{\partial t}+\nabla_{h} \cdot \mathbf{U}_{2}= & 0 .
\end{aligned}
$$

Here $\mathbf{U}_{i}, \mathbf{u}_{i}$, and $h_{i}$ denote the vertically averaged transport and velocity vectors, and the layer thicknesses of the upper $(i=1)$ and lower layer $(i=2)$ respectively, while $\eta_{1}$ represents the surface displacement, and $\eta_{2}$ the interface displacement.

Figure 14 shows the normalized horizontal velocity field (i.e., the velocity divided by $f R_{0}$ ) as a function of the normalized coordinates (i.e., $x / R_{0}$ and $y / R_{0}$ ) of three frontal eddies for four selected times $(t=6 T, t=9 T$, $t=12 T, t=15 T)$ defined, for $t=0$, by the same parameters characterizing the pulson simulated in the central experiment (see section 3). Here, however, the eddies evolve in a two-active-layer system having different water depths. The parameter $\delta$ expresses the ratio between the maximum eddy thickness and the water depth. Our simulations show that only for unrealistically large water depths the effect of an active ambient layer on the eddy dynamics is virtually absent (Fig. $14 ; \delta=$ $1 / 100)$. By decreasing water depths, the influence of the lower-layer dynamics becomes increasingly important. However, for values of $\delta$ typical of observed Gulf Stream rings (i.e., $1 / 20<\delta<1 / 6$ ) the reduced-gravity model describes warm-core eddies acceptably. By increasing $\delta$, the tendency for the simulated eddies to develop instabilities (Fig. 14; $\delta=1 / 3$ and $\delta=2 / 3$ ) rapidly increases. By $\delta=2 / 3$ the eddy coherence is destroyed shortly after $t=15 T$. These results are consistent with the results obtained by Cushman-Roisin et al. (1992), who investigated analytically the appropriateness of the reduced-gravity model for oceanic vortices on the $f$ plane as well as on the $\beta$ plane in the frame of frontal geostrophy.

\section{Discussion and conclusions}

In this study we investigated aspects of the decay of stable frontal warm-core eddies by using a new numerical layered frontal model capable of describing flow features characterized by the presence of a frontal line at the sea surface. The model was tested by comparing, for the first time, numerical and analytical solutions describing the temporal and spatial evolution of frontal, nonstationary warm-core eddies with paraboloidic shape and linear velocity field, the so-called pulsons. This step is necessary, as far as the numerical simulation of different phenomena related to the dynamics of frontal surface vortices is concerned. In fact, the credibility and utility of numerical primitive equation layered models in the description of frontal phenomena in general, and of frontal surface vortices in particular, depend significantly on their ability in reproducing isopycnic layer outcropping at the sea surface (Sun et al. 1993). But, surprisingly enough, such a comparison has never been performed before, in a nonstationary context, even in a qualitative way. Once the energy decay deriving from the numerical inaccuracies inherent in the techniques used for the solution of the discretized equations has been quantified and, as in our case, has been found to be small (once, i.e., it results that the lifetimes of simulated frontal eddies are larger than the lifetimes of most of the observed ones), the eddy decay induced by different dissipative processes can be investigated. We thus analyzed the effect of harmonic eddy diffusion, linear water entrainment, as well as linear and quadratic interfacial friction in inducing the eddy decay. Obviously, these parameterizations, which belong to the most commonly used ones in the simulation of oceanic processes carried out with layered models, are only crude representations of the real processes affecting the oceanic mesoscale flow field. Nevertheless they can help understanding the role played by different physical processes in the observed long-term evolution of oceanic flow features like warm-core eddies. The inclusion of harmonic eddy viscosity in our model can be used to assess the distance between analytical and numerical solutions, but it also serves as a parameterization of subgrid processes occurring in the vortex interior. Obviously, it is not intended to provide a realistic description of the complex processes occurring at the eddy surface front, nor it seems to us that it is possible to achieve such a description in the frame of a simple model like the one used in the present investigation. In fact, in order to achieve this goal, the three-dimensional flow structure at the eddy surface front should be accurately resolved. But, as stressed in our introduction, this task would require an unsustainably high computational effort. Simplified layer models sacrifice a detailed description of small-scale processes for the sake of a description of fundamental characteristics of largerscale processes (Cushman-Roisin 1987; Rogers 1989; Rubino et al. 1998a; Esenkov and Cushman-Roisin 1999). Moreover, according to previous work on frontal vortex dynamics (Csanady 1979), we included in our numerical model other parameterizations aimed at describing the interactions between frontal vortex and ambient ocean.

The role of water entrainment, independent of the specific parameterization used to take this process into account in layer models, is subtle. It in fact acts as an interfacial friction, but it also modifies the eddy dynamic equilibrium by changing the density difference between eddy and ambient layer. Our results indicate that it represents a minor term in the decay of the eddy energy. This result is in good agreement with the result obtained by Csanady (1979), who, however, considered this term as causing a mere mass transfer across the pycnocline separating a quiescent ocean from a frontal surface eddy in which the pulsating dynamics was neglected.

Also in good agreement with the results of Csanady 

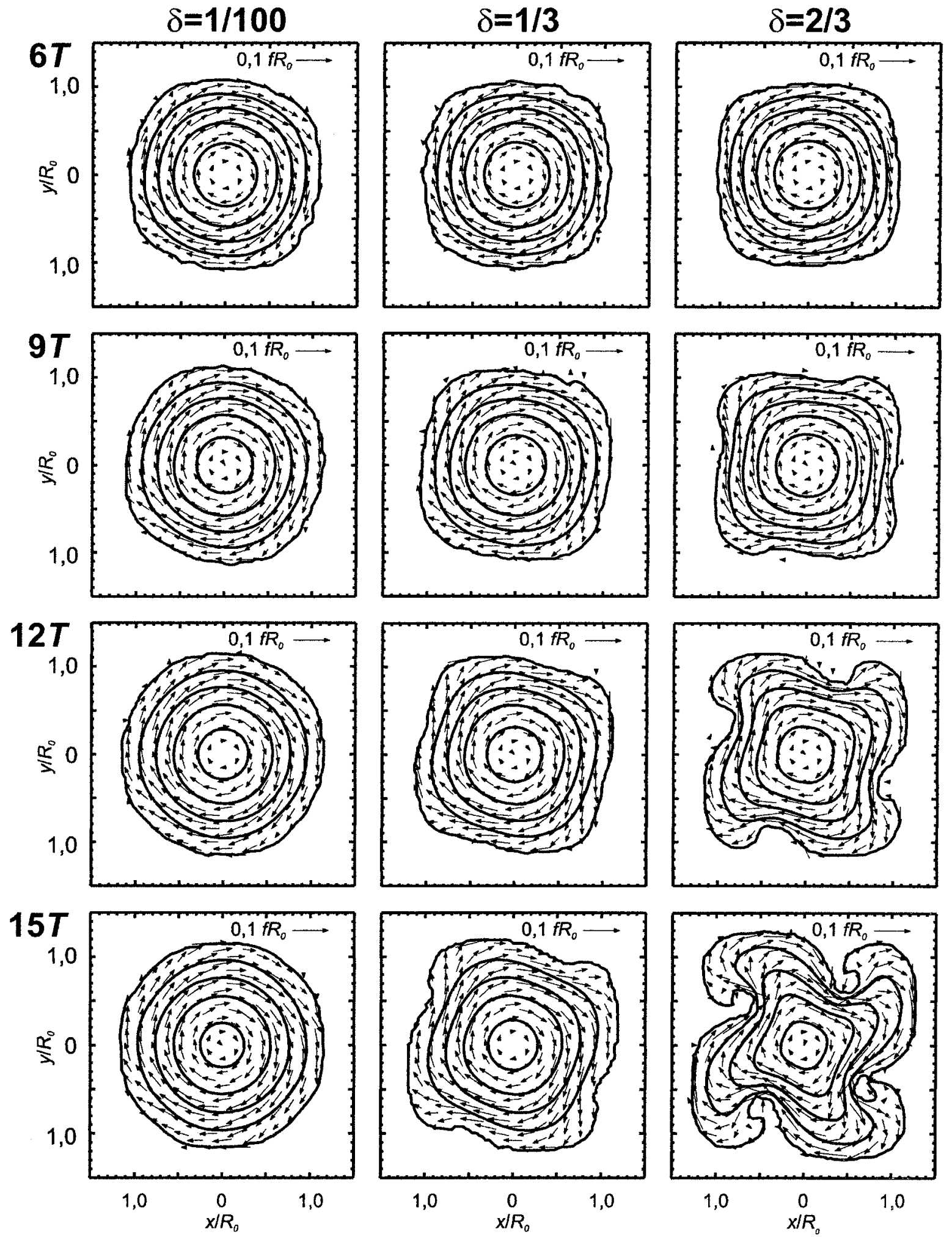

FIG. 14. Normalized horizontal velocity fields of three frontal eddies for four selected times $(t=6 T, t=9 T, t=12 T$, and $t=15 T)$ as simulated with the two-active-layer model. The parameter $\delta$ expresses the ratio between the maximum eddy thickness and the total water depth. For $t=0$ the eddies are defined by the same parameters characterizing the pulson simulated in the central experiment (see section 3). Note that also selected isolines of the simulated eddy thicknesses are presented. 
(the frames of whose work have been just indicated) is the fact that interfacial friction, instead, represents a much more effective mechanism for the eddy decay: The lifetimes of simulated eddies decrease, in fact, substantially, even by the inclusion of interfacial friction characterized by small drag coefficients. Choosing drag coefficients in the range typically used in the modeling of near-surface oceanic flows like warm core rings, results in lifetimes of the simulated features that are substantially shorter than those obtained in the inviscid case. But, what is even more interesting, the eddy inertial oscillations are in such a case substantially reduced. Thus, the results of our simulations suggest that interfacial friction is mainly responsible for the attenuation of the pulsating vortex dynamics, a phenomenon that, though foreseen analytically (Cushman-Roisin 1987; Rogers 1989; Rubino et al. 1998a) and observed in laboratory experiments (Ungarish and Huppert 1998), has never been observed in the real ocean. This, in turn, suggests that in a deep, stratified ocean (on the $f$ plane) the vortex decay due to radiation of the internal waves that would emerge from the vortex pulsation can be efficient only episodically. It is, in fact, conceivable that energy inputs to the eddy from the atmosphere or from the ambient ocean (in the form of wind stress curl providing momentum to the near-surface ocean, heat fluxes increasing the density contrast between surface layer and deep ocean, or vortex-current interactions) can rejuvenate the eddy pulsation dynamics from which internal, near inertial-wave radiation can occur.

The reduced-gravity assumption in the study of oceanic frontal vortices has a long tradition (Csanady 1979; Killworth 1983; Cushman-Roisin 1987; Pavia and Cushman-Roisin 1988; Pavia and Lopez 1994; Ochoa et al. 1998; Rubino et al. 1998a). It is, however, instructive to test the appropriateness of this parameterization in the specific context of surface frontal vortex dynamics obeying the nonlinear shallow-water equations on an $f$ plane in deep waters. To achieve this goal we compared the development of a surface frontal eddy evolving in a two-active-layer ocean having different water depths. Our results indicate that, although the effect of an active ambient layer on the vortex dynamics is virtually absent only for unrealistically large water depths, the differences between the results obtained using the reducedgravity model and the two-active-layer one become small for values of the ratio between maximum vortex thickness and total water depth typical for Gulf Stream rings. In this case, the simulated eddy lifetimes agree with observed ones that indicate that instabilities develop slowly. This behavior is consistent with the vortex evolution foreseen by an analytical theory developed by Cushman-Roisin et al. (1992) in the frame of frontal geostrophy.

\section{REFERENCES}

Andrews, J. C., and P. Scully-Power, 1976: The structure of an East Australian Current anticyclonic eddy. J. Phys. Oceanogr., 6, 756-765.
Backhaus, J. O., 1976: Zur Hydrodynamik im Flachwassergebiet. Ein numerisches Modell. Dtsch. Hydrogr. Z., 29, 222-238.

, 1985: A three-dimensional model for the simulation of the shelf sea dynamics. Dtsch. Hydrogr. Z., 38, 165-187.

Brandt, P., A. Rubino, W. Alpers, and J. O. Backhaus, 1997: Internal waves in the Strait of Messina studied by a numerical model and synthetic aperture radar images from the ERS-1/2 satellites. J. Phys. Oceanogr., 27, 648-663.

Burchard, H., 1995: Turbulenzmodellierung mit Anwendungen auf thermische Deckschichten im Meer und Strömungen in Wattengebieten. Report GKSS 95/E/30, GKSS-Forschungszentrum Geesthacht GmbH, Geesthacht, Germany, 299 pp.

Csanady, G. T., 1978: Turbulent interface layers. J. Geophys. Res., 83, 2329-2342.

1979: The birth and death of a warm core ring. J. Geophys. Res., 84, 777-780.

Cushman-Roisin, B., 1986: Frontal geostrophic dynamics. J. Phys. Oceanogr., 16, 132-143.

, 1987: Exact analytical solution for elliptical vortices of the shallow-water equations. Tellus, 39, 235-244.

__ , and S. Merchant-Both, 1995: Elliptical warm core rings in a two-layer ocean with ambient shear. J. Phys. Oceanogr., 25, 2011-2024.

_ _ G. G. Sutrin, and B. Tang, 1992: Two-layer geostrophic dynamics. Part I: Governing equations. J. Phys. Oceanogr., 22, $117-127$.

Esenkov, O. E., and B. Cushman-Roisin, 1999: Modeling of twolayer eddies and coastal flows with a particle method. J. Geophys. Res., 104, 10 959-10980.

Evans, R. H., K. S. Baker, O. B. Brown, and R. C. Smith, 1985: Chronology of warm-core ring 82B. J. Geophys. Res., 90, 88038812.

Garvine, R. W., 1982: A steady state model for buoyant surface plumes in coastal waters. Tellus, 34, 293-306.

__ 1984: Radial spreading of buoyant, surface plumes in coastal waters. J. Geophys. Res., 89, 1989-1996.

_ 1987: Estuary plumes and fronts in shelf waters: A layer model. J. Phys. Oceanogr., 17, 1877-1896.

—_, 1996: Buoyant discharge on the inner continental shelf: A frontal model. J. Mar. Res., 54, 1-33.

Hessner, K., 1998: Zur Dynamik mesoskaliger Frontenwirbel im Ozean. Shaker Verlag, $123 \mathrm{pp}$.

Joyce, T. M., 1984: Velocity and hydrographic structure of a Gulf Stream warm-core ring. J. Phys. Oceanogr., 14, 936-947.

Jungclaus, J. H., and J. O. Backhaus, 1994: Application of a transient reduced-gravity plume model to the Denmark Strait overflow. $J$. Geophys. Res., 99, 12 375-12 395.

Killworth, P. D., 1983: On the motion of isolated lenses on a betaplane. J. Phys. Oceanogr., 13, 368-376.

Matsuura, T., 1980: On the decay process of isolated, intense vortices in a two-layer ocean. J. Oceanogr. Soc. Jap., 36, 39-45.

_ 1995: The evolution of frontal-geostrophic vortices in a twolayer ocean. J. Phys. Oceanogr., 25, 2298-2318.

Neumann, G., and W. Pierson, 1966: Principles of Physical Oceanography. Prentice-Hall, 545 pp.

Nof, D., and L. M. Simon, 1987: Laboratory experiments on the merging of anticyclonic eddies. J. Phys. Oceanogr., 17, 343357.

Ochoa, J., J. Sheinbaum, and E. G. Pavia, 1998: Inhomogeneous rodons. J. Geophys. Res., 103, 24 869-24 880.

O'Donnell, J., 1988: A numerical technique to incorporate frontal boundaries in layer models of ocean dynamics. J. Phys. Oceanogr., 18, 1584-1600.

__ 1990: The formation and fate of a river plume: A numerical model. J. Phys. Oceanogr., 20, 551-559.

—_, and R. W. Garvine, 1983: A time-dependent, two-layer frontal model of buoyant plume dynamics. Tellus, 35, 73-80.

Olson, D. B., 1991: Rings in the ocean. Annu. Rev. Earth Planet. Sci., 19, 283-311. 
Pavia, E. G., and B. Cushman-Roisin, 1988: Modeling of oceanic fronts using a particle method. J. Geophys. Res., 93, 3554-3562. , and _ 1990: Merging of frontal eddies. J. Phys. Oceanogr., 20, 1886-1906.

, and M. López, 1994: Long-term evolution of elongated warm eddies. J. Phys. Oceanogr., 24, 2201-2208.

Rubino, A., P. Brandt, and K. Hessner, 1998a: Analytical solutions for circular eddies of the reduced-gravity, shallow-water equations. J. Phys. Oceanogr., 28, 999-1002.

_, S. Pierini, and J. O. Backhaus, 1998b: Dispersive mudslideinduced tsunamis. Nonlinear Proc. Geophys., 5, 127-136.
Rogers, C., 1989: Elliptic warm-core theory: The pulsrodon. Phys. Lett., 138, 267-273.

Saunders, P. M., 1971: Anticyclonic eddies formed from shoreward meanders of the Gulf Stream. Deep-Sea Res., 18, 1207-1220.

Stegner, A., and D. G. Dritschel, 2000: A numerical investigation of the stability of isolated shallow water vortices. J. Phys. Oceanogr., 30, 2562-2573.

Sun, S., R. Bleck, and E. P. Chassignet, 1993: Layer outcropping in numerical models of stratified flows. J. Phys. Oceanogr., 23, 1877-1884.

Ungarish, M., and H. E. Huppert, 1998: The effect of rotation on axisymmetric gravity currents. J. Fluid Mech., 362, 17-51. 